\title{
Quels apports de la littérature pour comprendre et construire la transition agroécologique en viticulture?
}

\author{
Francis Macary $^{1,}{ }^{*}$, Fiona Guerendel ${ }^{2}$ et Adeline Alonso Ugaglia ${ }^{3}$ \\ ${ }^{1}$ INRAE, UR ETBX, 33612 Cestas, France \\ 2 INRAE, UR ETBX, Bordeaux Sciences Agro, Univ. Bordeaux, 33612 Cestas, France \\ ${ }^{3}$ INRAE, UMR SAVE, Bordeaux Sciences Agro, Univ. Bordeaux, 33140 Villenave d'Ornon, France
}

\begin{abstract}
Résumé - En s'appuyant sur une revue de la littérature, l'article propose un état de l'art centré sur l'agroécologie, concept polysémique, afin d'en éclairer la portée pour la viticulture et son application, dans le cadre d'un projet de recherche destiné à comprendre et construire la transition agroécologique dans les vignobles. L'agroécologie est tour à tour considérée comme une discipline scientifique proposant une analyse systémique en intégrant des sciences humaines et sociales, un ensemble de pratiques intégrant les principes de l'écologie en agronomie (travailler avec la nature), ou un mouvement social. L'échelle d'analyse a progressivement été élargie depuis le niveau de la parcelle agricole vers celui des territoires, jusqu'à des approches globales du système alimentaire. La vigne est une monoculture pérenne pour laquelle les enjeux de la transition agroécologique sont particulièrement importants eu égard notamment à la forte pression des pesticides appliqués en vue de la protection phytosanitaire. Ce débat, devenu sociétal, implique une évolution des systèmes de production viticole afin de satisfaire les enjeux environnementaux et de santé publique. Pour construire et évaluer des scénarios de transition agroécologique auprès des professionnels de la filière, nous avons réalisé une revue de la littérature qui a pu mettre en évidence différentes pratiques agroécologiques dans les vignobles : préservation de la biodiversité, baisse de l'usage des pesticides par une régulation des bioagresseurs et des adventices, conservation de la qualité des sols, adaptation au changement climatique. Elle permet également de discuter des enjeux d'élargissement de l'acception du concept d'agroécologie dans le contexte actuel. Ces éléments sont essentiels à l'identification de critères d'évaluation pour la construction de scénarios d'évolution des modes conventionnels de production viticole dans le cadre de la transition écologique.
\end{abstract}

Mots clés : agroécologie / agroécosystèmes / biodiversité / pratiques agricoles / viticulture / vignoble

Abstract - What contributions from the literature to understand and build the agroecological transition in viticulture? Based on a review of the literature, the article proposes a state of the art centered on agroecology, a polysemic concept, in order to shed light on its scope for viticulture and its application within the framework of a project research aimed at understanding and building the agroecological transition in vineyards. Agroecology is considered alternately as a scientific discipline offering a systemic analysis by integrating human and social sciences, as a set of practices integrating the principles of ecology in agronomy (working with nature), or as a social movement. The scale of analysis has gradually been broadened from the level of agricultural plots to the level of territories, up to comprehensive approaches to the food system. Vine is a perennial monoculture for which the challenges of agroecological transition are particularly strong, in view of the strong pressure of pesticides applied for phytosanitary protection. This debate, which has become societal, implies an evolution of wine production systems in order to meet environmental and public health issues. In order to be able to construct and evaluate agroecological transition scenarios with professionals in the sector, we carried out a review of the literature which highlighted various agroecological practices in vineyards: preservation of biodiversity, reduction in the use of pesticides by regulation of pests and weeds, conservation of crop quality soils.. It also provides an opportunity to discuss the issues of broadening the meaning of the concept of agroecology in the current context. These elements are essential for identifying evaluation criteria in scenarios construction for the evolution of wine production conventional methods, as part of the ecological transition.

Keywords: agroecology / agroecosystems / biodiversity / agricultural practices / viticulture / vineyard

\footnotetext{
*Auteur de correspondance : francis.macary@inrae.fr
} 


\section{Introduction}

Dans les pays occidentaux, le modèle agricole productiviste mis en œuvre après la seconde guerre mondiale avait pour objectif majeur d'assurer l'autonomie alimentaire, la protection sanitaire des aliments et de permettre de réduire au mieux la part du budget dédiée à l'alimentation (Parmentier, 2007). En trois décennies, ces objectifs ont été atteints, voire dépassés, mais à un coût environnemental très élevé (pollution des eaux de surface, des eaux souterraines, des sols, chute de la biodiversité) (Aubertot et al., 2005; Barriuso, 2004) et avec des impacts pour la santé humaine, notamment en rapport avec l'usage de produits phytosanitaires (Alavanja et al., 2004; Aurias et al., 2016; Baldi et Lebailly, 2007 ; Baldi et al., 2012). De plus, l'agriculture conventionnelle basée sur l'usage d'intrants relevant de la chimie de synthèse (fertilisants, pesticides) est peu ouverte à la société, ce qui induit une forme de désapprobation par celle-ci et une forme de rejet des produits phytosanitaires et de leurs conséquences sur les écosystèmes et la santé humaine, désormais avérées (INSERM, 2013; Aurias et al., 2016; Garrigou et al., 2020). Ce mode conventionnel de production agricole, de plus en plus décrié, a progressivement évolué vers des démarches raisonnées, notamment en termes d'usage d'intrants (Doré et al., 2008). Mais pour autant, on est encore loin de la rupture nécessaire pour réconcilier production agricole et écosystèmes connexes (Tardieu, 2012). Les écosystèmes sont également porteurs de facteurs de progrès considérables en termes de biodiversité, de protection des ravageurs et de qualité des sols, pour peu que les systèmes de conduite prennent en compte et préservent, voire favorisent, les services rendus (Altieri, 1995). Le grand principe fondateur de l'agroécologie consiste à valoriser la qualité des écosystèmes dans l'acte de production agricole (Doré et Bellon, 2019). Dans cet ouvrage, les auteurs décrivent l'évolution lente et progressive des mentalités dans le monde agricole occidental en faveur de l'agroécologie.

Les externalités négatives de l'agriculture sont amplifiées dans les systèmes de monoculture pérenne telle que la vigne, particulièrement sensible aux pathogènes celle-ci représente une part importante des produits phytosanitaires utilisés par rapport à la surface dédiée dans un pays comme la France $(3 \%$ de la surface agricole utile, $30 \%$ du tonnage global) (Gary et al., 2009). Les enjeux de la transition agroécologique (MAAF, 2012) sont donc prégnants pour cette filière. La notion de transition fait référence à un processus complexe qui modifie radicalement les principes d'un système en place, ce qui engendre des changements dans les valeurs des acteurs socio-professionnels ainsi que dans les techniques et pratiques qu'ils mobilisent (Hazard et al., 2017). La transition agroécologique désigne alors un changement de modèle agricole et s'appuie sur les savoirs en agroécologie, sur l'engagement des acteurs de la filière de production pour appliquer ces savoirs sur le territoire, et sur la territorialisation de l'agriculture en assurant une bonne connexion entre production agricole et consommation (Hazard et al., 2017).

La littérature internationale s'est pourtant relativement peu intéressée à l'identification de pratiques et de trajectoires de transition agroécologique pour la filière viticole. Dans le contexte d'un projet de recherche visant à une réduction drastique de l'utilisation des produits phytosanitaires en viticulture, nous avons pour objectifs la construction et l'évaluation de scénarios d'évolution des modes de production et des pratiques, orientés vers une démarche systémique en agroécologie. Pour cela nous avons été amenés à réaliser la présente analyse de la littérature. La démarche s'est appuyée, d'une part, sur un état de l'art sur l'agroécologie, ayant fait l'objet de publications scientifiques, pour appréhender et mobiliser les concepts et les connaissances inhérentes pour la viticulture; d'autre part, sur un recensement des initiatives agroécologiques existant dans les pratiques viticoles.

Cet article présente d'abord une synthèse des concepts et approches de l'agroécologie mobilisables en viticulture. Puis nous évoquons les démarches et les pratiques clés pour la mise en œuvre de la transition agroécologique en viticulture. La dernière partie discute enfin de la nécessité d'une conception plus large de l'agroécologie pour répondre aux enjeux sociétaux auxquels est confrontée la viticulture dans le contexte actuel.

\section{Une évolution des concepts de l'agroécologie et de ses spécificités pour la viticulture}

La notion d'agroécologie a évolué dans le temps et géographiquement dans les différents pays où elle a été étudiée et où on se l'est appropriée. Trois périodes peuvent être considérées dans l'évolution du concept: la période scientifique initiale (1930-fin des années 1960), la deuxième période dite d'expansion de l'agroécologie sur le terrain (1970-années 2000), et une période récente au cours de la dernière décennie avec une démarche plus politique.

Dans les pays du Sud, l'agroécologie est d'abord apparue comme un mouvement social dans un cadre militant pour l'accès au foncier (par exemple Paysans sans terre au Brésil) et comme moteur pour la recherche de pratiques agricoles plus écologiques. Dans les pays occidentaux, elle a été perçue initialement comme une discipline scientifique (États-Unis, Allemagne) ou comme un ensemble de pratiques agricoles écologiques (France), avant d'y incorporer des aspects de sciences humaines, éthiques, philosophiques, etc. (Wezel et al., 2009) (Fig. 1). Dans les différents pays, il existe très souvent un lien entre la vision politique de l'agroécologie (le mouvement social), le moyen de produire du savoir sur la discipline et les applications technologiques (les pratiques) pour atteindre le but recherché (la science) (Wezel et al., 2009; Francis et al., 2003).

La question de l'engagement du secteur viticole dans une transition agroécologique est récente compte-tenu de l'évolution du concept. Elle a émergé dans les années 2004-2010 dans les travaux des scientifiques (Nicholls et al., 2004; Tisseyre et al., 2007).

\subsection{Période initiale (1930-1970) : une approche scientifique}

La première évocation de l'agroécologie dans la littérature scientifique date des années 1930 (Calame, 2016). En 1928, l'agronome américain d'origine russe Bensin évoque l'agroécologie dans un article scientifique portant sur une approche de 


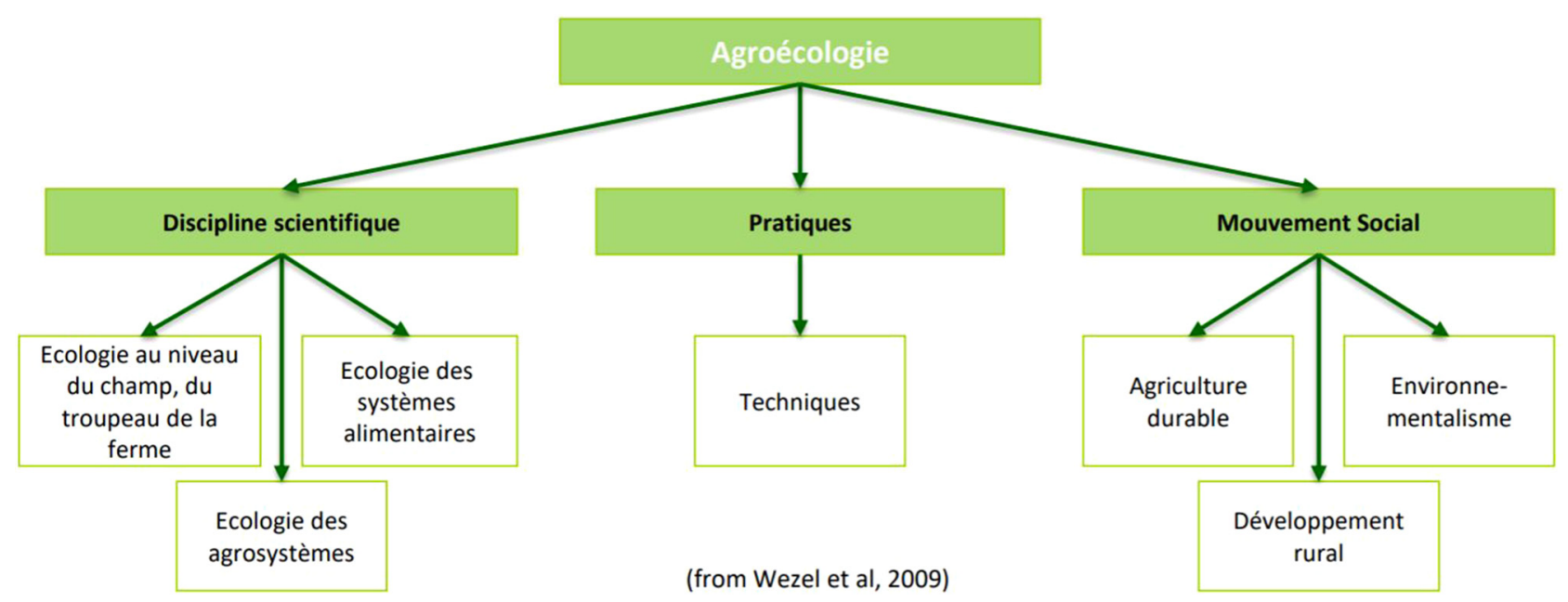

Fig. 1. Schéma conceptuel de l'agroécologie (d'après Wezel et al., 2009).

Fig. 1. Conceptual diagram of agroecology (from Wezel et al., 2009).

l'agronomie s'inspirant des acquis de l'écologie pour la production alimentaire, en prenant appui sur les contextes locaux plus que sur des solutions généralistes (Doré et Bellon, 2019). Durant cette période, le terme «agroécologie» est surtout utilisé pour parler d'une science, de l'inclusion de l'écologie dans l'agriculture, et de l'évolution de deux disciplines (écologie et agronomie) vers une seule, qui étudie les interactions biotiques au sein des agroécosystèmes. L'agronome français Hénin (1967) établit la relation avec l'agronomie de la façon suivante: «l'agronomie est de l'écologie appliquée à la production de plantes cultivées et la gestion de terres agricoles ». Le système analysé est restreint à la parcelle.

\subsection{Deuxième période (1970-2010) : l'expansion du concept sur le terrain}

L'agroécologie, discipline scientifique, évolue ensuite de manière différenciée dans le temps et suivant les pays, pour devenir également un mouvement social et un ensemble de pratiques agricoles. Dans le monde scientifique, elle gagne en popularité pendant les années 1960. Depuis les années 1980, l'agroécologie a émergé en tant que cadre d'analyse reposant sur des méthodes holistiques afin de protéger les ressources naturelles et de gérer des agrosystèmes de manière durable. Francis et al. (2003) et Gliessman (1998, 2015) présentent cette évolution comme une réponse à la révolution verte qui a provoqué une intensification et une spécialisation accrue des agroécosystèmes. La considération de l'agroécologie comme un mouvement social n'a été introduite qu'au début des années 1990, surtout en Amérique latine, et a été étudiée aux ÉtatsUnis par des auteurs tel qu'Altieri (1995). L'agroécologie définit alors non seulement une nouvelle façon d'envisager l'agriculture, mais aussi une nouvelle manière de définir la relation de l'agriculture avec la société. L'échelle d'étude devient donc moins centrée sur la parcelle agricole et s'étend sur tout le réseau alimentaire, prenant en compte les producteurs et les consommateurs comme acteurs de cette agriculture (Francis et al., 2003; Wezel et al., 2009). Le concept d'agroécologie montre ainsi que les pratiques agricoles et le système alimentaire ne peuvent pas être dissociés puisqu'ils dépendent tous les deux du contexte naturel et socio-économique dans lequel ils s'insèrent (Hatt et al., 2016).

\subsection{Depuis les années 2010 : agroécologie et enjeux de société}

De Schutter (2010), rapporteur des Nations unies pour le droit à l'alimentation, considère que l'agroécologie représente un modèle alternatif pouvant répondre aux crises économiques, sociales et écologiques en conciliant activités humaines et ressources planétaires. Plus récemment, en France, le ministre de l'Agriculture lance en 2012 le Projet agroécologique pour la France (MAAF, 2012). Ce projet cible le retour de l'agronomie au cœur des processus de production, la prise en compte de l'écologie afin de valoriser au mieux les agroécosystèmes et la transition agroécologique, tout en précisant l'impératif de concilier performances environnementale et économique des productions agricoles. Ce concept est inscrit dans la Loi d'avenir agricole (JORF, 2014). L'agroécologie est alors introduite dans la sphère sociétale via les institutions (Calame, 2016). Rahbi (2015), paysan écrivain conférencier, va plus loin et considère que l'agroécologie, outre son rôle nourricier en respectant les agroécosystèmes et la santé humaine par la qualité alimentaire produite, est aussi une éthique de vie, c'est-à-dire qu'elle offre la possibilité «d'élaborer les cultures et toutes les activités qui constituent les sociétés ».

Le concept d'agroécologie est ainsi polysémique, et l'on en trouve de multiples acceptions dans la littérature qui débouchent sur différentes définitions (Calame, 2016) (Tab. 1). Celles-ci relèvent de différents courants :

- l'agroécologie des systèmes productifs, reposant sur l'application des principes de l'écologie à l'agriculture (Altieri, 1995). Elle a débouché en agronomie sur un ensemble de méthodes et de pratiques, socle d'une révision des liens entre agriculture et écosystèmes dont le but est de garantir la préservation des ressources naturelles; 
Tableau 1. Diversité des définitions de l'agroécologie dans la littérature.

Table 1. Diversity of definitions of agroecology in the literature.

\begin{tabular}{ll}
\hline Références & Quelques définitions de l'agroécologie \\
\hline Francis et al., 2003 & $\begin{array}{l}\text { L'agroécologie représente «l'étude intégrative de l'écologie de l'ensemble des systèmes alimentaires, } \\
\text { englobant les dimensions écologiques, économiques et sociales - ou plus simplement l'écologie des } \\
\text { systèmes alimentaires » }\end{array}$ \\
& L'agroécologie est une alternative à une agriculture intensive qui promeut des systèmes de production \\
& agricole valorisant la diversité biologique et les processus naturels (cycles de l'azote, du carbone, de \\
l'eau, équilibres biologiques entre organismes ravageurs et auxiliaires des cultures...). Elle se développe & dans le champ scientifique comme une approche intégrant les concepts et méthodes d'une diversité de \\
& disciplines, dont l'agronomie, l'écologie, l'économie, la sociologie \\
& Visant à promouvoir les services rendus par les processus naturels, elle analyse à différents niveaux (de \\
& la parcelle au territoire, de l'individu à la communauté, du court au long terme) les relations évolutives \\
& qui se créent au sein de ces systèmes entre le vivant, son mode de gestion et le contexte écologique, \\
& économique et social de cette gestion
\end{tabular}

Baret, 2017

«L'agroécologie est un ensemble de principes d'action fondés sur deux grandes idées. La première: on travaille avec la nature et non pas contre elle. C'est donc une agriculture qui s'appuie sur le fonctionnement naturel d'un écosystème. La seconde : l'agriculture doit être efficace d'un point de vue économique - le but étant de gagner sa vie et nourrir sa famille - c'est au niveau du système qu'il faut réfléchir et non plus au niveau de la parcelle»

FAO, 2018a

L'agroécologie est une discipline scientifique, un ensemble de pratiques, un mouvement social. Sur un plan scientifique, la question est de savoir comment les différentes composantes de l'agroécosystème interagissent. Concernant les pratiques, elle recherche des systèmes agricoles durables qui optimisent et stabilisent les rendements. En tant que mouvement social, elle assume des rôles multifonctionnels pour l'agriculture, promeut la justice sociale, nourrit l'identité et la culture et renforce la viabilité économique des zones rurales. L'agroécologie est une approche intégrée qui applique simultanément des concepts et des principes écologiques et sociaux à la constitution et à la gestion de systèmes agroalimentaires

Côte et al., 2018

Les auteurs font référence à la notion d'agroécologie faible, qui rétablit dans les exploitations et les agrosystèmes le bouclage des cycles biologiques (carbone, azote) pour réduire l'utilisation des produits phytosanitaires. Mais ce modèle n'interpelle ni l'organisation de la recherche, ni celle du marché. Les auteurs évoquent également la notion d'agroécologie forte, qui amène à une réforme générale du système alimentaire pour le rendre plus durable, à une recherche participative, à une consommation responsable et à un renouvellement des politiques publiques conduisant à une régulation des marchés et à l'autonomie et la responsabilisation des collectivités locales.

Doré et Bellon, 2019

Pour Altieri, «l'agroécologie est une application des principes de l'écologie à l'agriculture», définition initiale de l'agroécologie, inspirée des travaux de Bensin dans les années 1930

- l'agroécologie des systèmes alimentaires, vue comme un programme interdisciplinaire support d'un développement agricole et alimentaire durable (Duru et al., 2014; Hazard et al., 2017);

- l'agroécologie comme projet de société (Rahbi, 2015).

\subsection{Spécificités de l'agroécologie pour la viticulture}

De façon plus spécifique en viticulture, Gary et al. (2017) ont mis en évidence tout l'intérêt du développement les vignobles agroécologiques alliant innovations de gestion et aménagement du territoire à l'échelle des parcelles, des exploitations et des paysages. En France, l'Institut national des appellations d'origine (INAO) et l'Institut français de la vigne et du vin (IFV) ont proposé en 2017 une liste des bonnes pratiques agroécologiques (Lempereur et al., 2017), dont certaines relèvent de propositions déjà édictées dans le cadre d'un guide de conception de systèmes de production en viticulture afin de réduire les apports et impacts de pesticides (INRA et al., 2011). Shields et al. (2016) présentent les résultats d'un travail en Nouvelle-Zélande sur les espèces végétales endémiques à faible croissance, évaluées pour leurs avantages potentiels en tant qu'unités de prestation de services ou fournisseurs de services écosystémiques. Garcia et al. (2019) ont étudié l'impact des stratégies de gestion des sols et des apports des racines caractéristiques des communautés végétales et du carbone organique du sol pour expliquer la stabilité des agrégats du sol dans les vignobles. D'autres travaux prennent en compte les parties prenantes dans la construction du concept. Par exemple, Nicholls et al. (2004) présentent une méthodologie pratique en agroécologie pour évaluer rapidement la qualité des sols et la santé des cultures des systèmes viticoles à l'aide d'indicateurs simples, choisis, appliqués et interprétés conjointement par agriculteurs et les chercheurs. Warner (2007) évoque une relation entre la qualité 
des vins attendue par les consommateurs en Californie et une baisse de la pression phytopharmaceutique sur le vignoble. Ces travaux, proches de l'acception de Côte et al. (2018), se concentrent essentiellement sur les agroécosystèmes viticoles, i.e. les systèmes de production viticole et leur rapport aux ressources naturelles et aux services rendus.

Toutefois, il reste à interroger les fondamentaux de la transition agroécologique dans les exploitations viticoles.

\section{L'agroécologie, des démarches et des pratiques récentes dans les vignobles}

La vigne est une production à fort enjeu sociétal, comptetenu notamment de sa dépendance aux produits pesticides pour la protection phytosanitaire. En tant que production pérenne, elle ne peut pas bénéficier de pratiques adaptées aux systèmes de grande culture, telles que les rotations culturales qui permettent par exemple de réduire l'impact des adventices. En revanche, elle bénéficie d'autres d'atouts liés à la pérennité, tels que la possibilité de favoriser les habitats d'auxiliaires, d'assurer une gestion des sols permettant de favoriser la matière organique et la faune tellurique.

La littérature fait ressortir les grands enjeux de la conception d'agroécosystèmes en adéquation avec les principes fondateurs de l'agroécologie, que nous discutons au regard des spécificités de la viticulture.

\subsection{Préservation et développement de la biodiversité}

La préservation de la biodiversité des espèces végétales et animales va de pair avec les services écosystémiques qui y sont liés (Altieri, 1999), tels que la régulation naturelle des bioagresseurs (Brévault et Clouvel, 2019), la pollinisation et la santé des sols, services dont dépend la production (FAO, 2018b). Dans une méta-analyse, Letourneau et al. (2009) ont révélé que, dans $71 \%$ des études explicitant la relation entre la richesse spécifique et le contrôle de populations d'arthropodes herbivores, la relation établie est positive, d'autant plus dans les systèmes agricoles. En Nouvelle-Zélande, des espèces végétales endémiques à faible croissance ont été évaluées au vignoble pour leur rôle dans la suppression des mauvaises herbes, la conservation des invertébrés utiles (arthropodes), le maintien de l'humidité du sol, ainsi que la rétention et le développement de l'activité microbienne (Shields et al., 2016). Les éléments du paysage (arbres, bosquets, bordures de parcelle, talus, fleurs, cours d'eau, étangs) ont également été reconnus comme refuges de biodiversité faunistique et floristique (INRA et IFV, 2017a). Ainsi, les principes de préservation et de développement de la biodiversité sont à privilégier dans la conception de systèmes viticoles agroécologiques :

- aménagement de surfaces écologiques de compensation : bandes enherbées, voire bandes fleuries en bordure de champs dans les tournières et les espaces inter-parcellaires, enherbement des talus, fossés, des chemins aux abords des parcelles viticoles, implantation de haies afin de fournir des habitats aux divers auxiliaires (Baudry et al., 2000);

- absence d'application de fertilisants ou de produits phytosanitaires sur les zones écologiques végétalisées ;

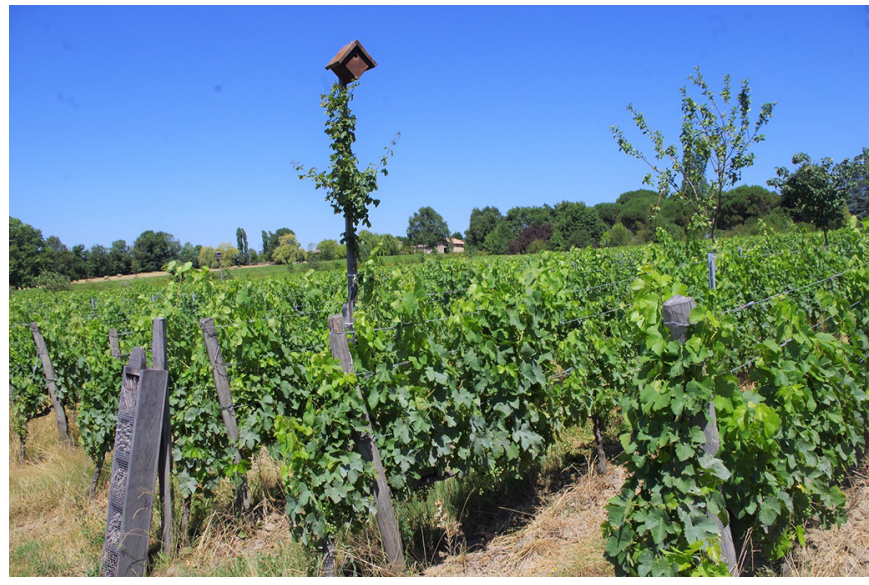

Fig. 2. L'agroforesterie et la biodiversité animale au Domaine Emile Grellier (F. Macary).

Fig. 2. Agroforestry and animal biodiversity at Domaine Emile Grellier (F. Macary).

- développement de l'agroforesterie dans le vignoble afin de briser la monoculture de la vigne, d'éviter les discontinuités paysagères et de favoriser les habitats d'auxiliaires aux prédateurs (oiseaux, chauves-souris qui consomment les vers de la grappe) (Fig. 2).

\subsection{Réduction des pesticides par une régulation des bioagresseurs et techniques alternatives}

Parmi les produits phytosanitaires de synthèse utilisés en viticulture dite conventionnelle (herbicides, fongicides, insecticides) pour la régulation des bioagresseurs des cultures, certains classés CMR (cancérigène, mutagène, reprotoxique) et $\mathrm{PE}$ (perturbateurs endocriniens) ont des répercussions avérées sur la santé humaine et sur la biodiversité environnante (Baldi et Lebailly, 2007). Cependant, des alternatives à l'usage de ces molécules existent. Il s'agit par exemple des méthodes dites de biocontrôle ou de lutte biologique contre les ravageurs, c'est-à-dire des méthodes de contrôle des populations reposant sur des mécanismes naturels (Gary et al., 2017). La lutte biologique par conservation des habitats favorise les populations d'auxiliaires afin de contrôler les populations de prédateurs. Pour pérenniser l'activité de ces auxiliaires, des aménagements favorables à leur développement sont nécessaires dans ou autour des parcelles : bandes fleuries, couverts herbacés, haies (Cervek, 2012). La conception et la gestion des systèmes viticoles doivent prendre en compte l'arrangement des végétaux dans le temps et l'espace en contexte de monoculture, la composition et l'abondance de la végétation non-productrice dans et autour des parcelles, le type de sol, l'environnement, ainsi que le mode de production mis en œuvre (INRA et al., 2011; Caquet et al., 2020) (Fig. 3). Il est cependant difficile de prédire avec précision les dynamiques des ravageurs.

D'autres méthodes de lutte biologique reposent sur la perturbation des cycles de reproduction des insectes. La confusion sexuelle consiste à diffuser dans l'atmosphère une 


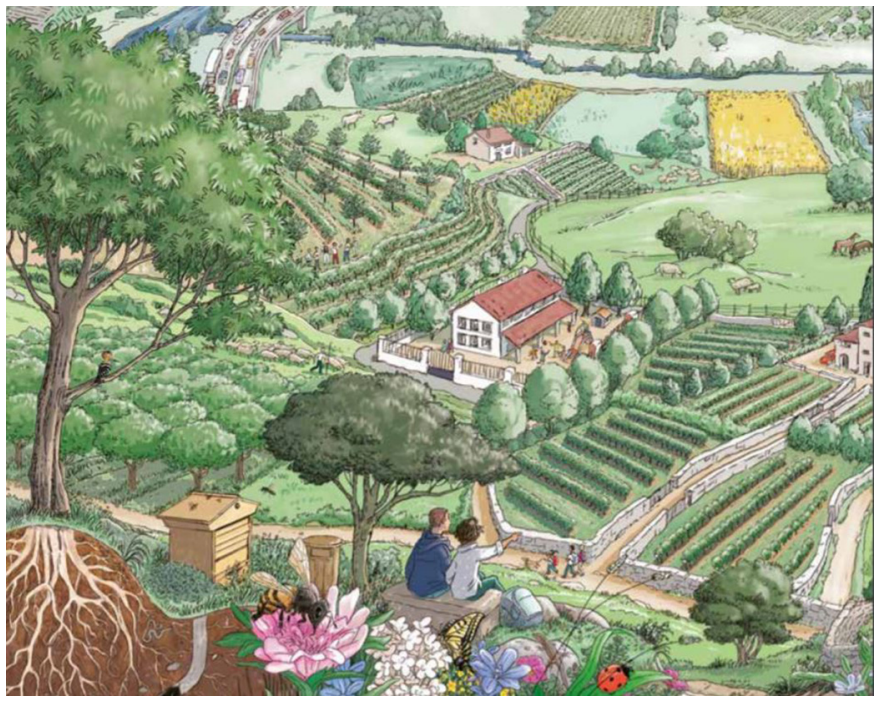

Fig. 3. Illustration d'un paysage viticole type (Lempereur et al., 2017).

Fig. 3. Illustration of a typical wine landscape (Lempereur et al., 2017).

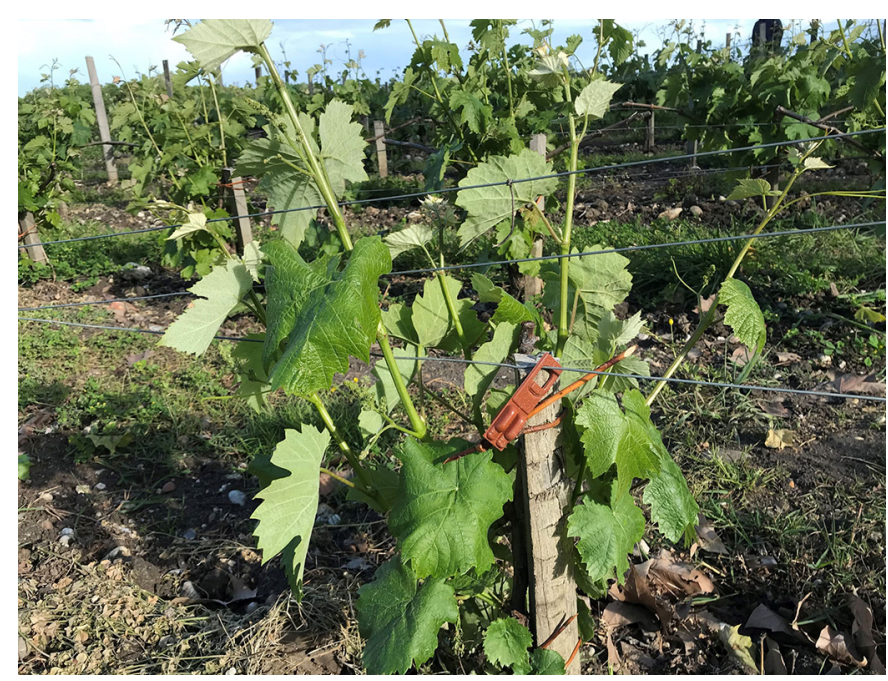

Fig. 4. Capsules de phéromones pour maîtriser les vers de la grappe (F. Macary).

Fig. 4. Pheromone capsules to control cluster worms (F. Macary).

quantité de phéromones synthétiques qui perturbent la rencontre entre mâles et femelles, et empêchent ainsi l'accouplement. Elle est principalement utilisée pour lutter contre les vers de la grappe tels que Cochylis (Eupoecilia ambiguella) et Eudémis (Lobesia botrana) (Fig. 4) en viticulture (INRA et IFV, 2017). Concernant la lutte contre les adventices, le désherbage des inter-rangs et du cavaillon (zone comprise sous le rang) est conduit en systèmes biologiques et agroécologiques de façon mécanique, soit par tonte soit par des outils de travail du sol superficiels (INRA et IFV, 2017). L'enherbement est privilégié (Fig. 5). C'est donc l'ensemble de la protection du vignoble qui est requestionné, en relation avec

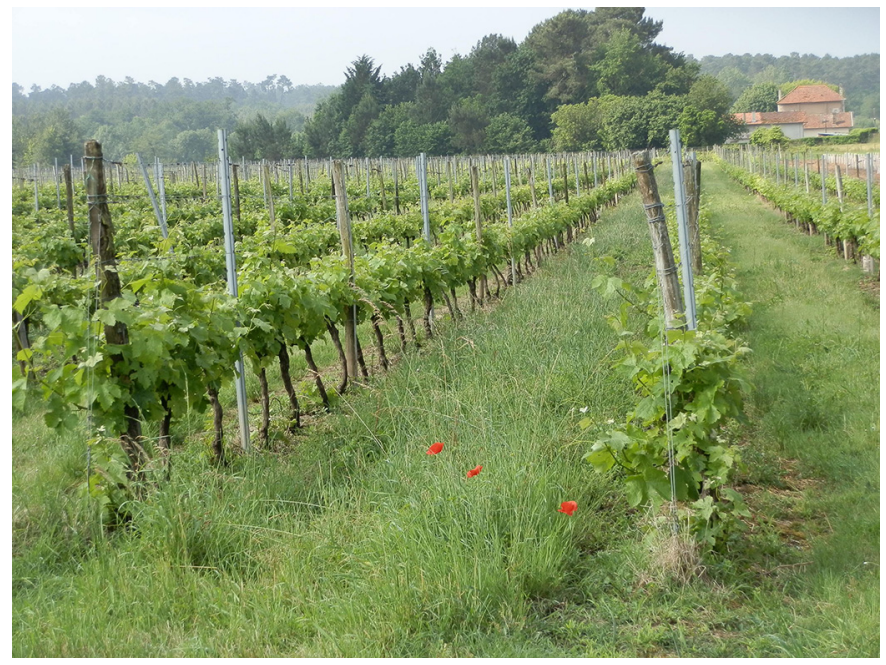

Fig. 5. Biodiversité végétale dans le vignoble (F. Macary).

Fig. 5. Plant biodiversity in the vineyard (F. Macary).

l'écosystème des parcelles et leur biodiversité associée, à l'aune des principes de l'agroécologie.

La création de cépages résistants aux cryptogames de la vigne les plus importants - mildiou (Plasmopara viticola) et oïdium (Erysiphe necator) - avait débuté dans les années 1950 avec des hybrides producteurs directs, résultants du croisement entre vignes américaines résistantes (Vitis rupestris, Vitis lincecumi, Vitis berlandieri) et vigne européenne sensible (Vitis vinifera). Mais leur qualité médiocre sur un plan œnologique avait conduit à leur interdiction en zone d'Appellation d'origine contrôlée (AOC) et à une forte régression en vin de table. À partir de 1974, le chercheur Alain Bouquet a conduit des travaux pour incorporer à la vigne européenne des facteurs de résistance provenant de l'espèce Vitis rotundifolia. Ce programme a permis d'aboutir à des variétés monogéniques «Bouquet», portant un gène de résistance au mildiou et un à l'oïdium. Depuis 2000, l'INRA a engagé un programme de création variétale (nommé ResDur) à partir des variétés «Bouquet», qui vise un double objectif: accroître la durabilité des résistances de cépages monogéniques au mildiou et à l'oïdium par intégration de plusieurs gènes de résistance et améliorer leurs qualités agronomiques, technologiques et gustatives. Ce programme, conduit en partenariat avec l'IFV depuis 2012, a permis l'inscription au catalogue officiel des raisins de vins de cuve des premières variétés telles qu'Artaban, Vidoc, Floréal, Voltis (INRA et IFV, 2017). Ces variétés présentent une résistante totale à l'oïdium et forte au mildiou, mais quelques traitements phytosanitaires sont encore nécessaires pour assurer une protection totale pour ce pathogène. De plus, la réglementation actuelle pour les vins d'appellation en France fixe une limite quantitative à un volume maximum de $5 \%$ de vins issus de cépages résistants au sein d'un assemblage.

\subsection{Conservation de la qualité et nutrition des sols}

L'utilisation de produits phytosanitaires a appauvri, voire parfois détruit progressivement les sols cultivables (Aubertot et al., 2005). Le retournement systématique des sols (par action 
du labour) ainsi que l'emploi de pesticides ont peu à peu détruit la vie biologique du sol. En plus d'être une composante essentielle d'un écosystème agricole, le sol fournit une multitude de services écosystémiques, dont la séquestration de carbone et la régulation du climat. Les sols, y compris en viticulture, ont longtemps été considérés comme un support quasi inerte et non pas comme un milieu biologique actif et nourricier pour les plantes et facteur de résistance aux agressions cryptogamiques (Bourguignon, 2017). Le maintien de la structure du sol pose également le problème des conséquences des processus érosifs et de la lixiviation des éléments nutritifs, ainsi que la régulation de l'abondance des organismes vivants telluriques (Henneron et al., 2015).

L'agriculture de conservation est un type d'agriculture qui vise à réduire, voire à supprimer totalement le travail du sol, par la mise en place d'une couverture permanente et de successions culturales diversifiées (Schaller, 2013). L'amélioration de la vie biologique des agrosystèmes engendre alors un accroissement de la matière organique dans le sol, un stockage de carbone, une évaporation réduite et ainsi un meilleur maintien hygrométrique. L'agriculture de conservation est basée sur des grands principes qui doivent être appliqués en même temps (Schaller, 2013). Ainsi en viticulture:

- la réduction, voire la suppression, du labour et son remplacement par des techniques de travail du sol moins agressives pour le maintien de la biodiversité dans le sol cultivé. En viticulture, un système agroécologique favorisera le non-labour;

- la couverture du sol qui permet de recycler la matière, d'améliorer sa structure, d'enrichir sa biomasse, augmentant in fine la proportion de matière organique dans le sol. Le développement de systèmes agroforestiers apporte une forte contribution à la séquestration du carbone. (Albrecht et Kandji, 2003). De plus, cette couverture limite le développement des adventices et donc l'usage des herbicides, dont le glyphosate. Des plantes de couverture peuvent aussi être utilisées en interculture des rangs de vigne et les résidus laissés au sol (mulching). La retenue hydrique au niveau du sol est alors meilleure, ce qui augmente la résistance au stress hydrique de la vigne dans un contexte de changement climatique. Les agrégats dans le sol sont aussi plus stables, de par cette couverture protectrice. Les sols sont donc plus résistants face à des phénomènes comme la lixiviation des éléments minéraux (Mulumba et Lal, 2008).

\subsection{Adaptation du vignoble au changement climatique}

Le dernier rapport du Groupe d'experts intergouvernemental sur l'évolution du climat (GIEC) précise que l'accroissement des gaz à effet de serre, principalement du dioxyde de carbone, aura pour effet d'augmenter la température terrestre de $1,4^{\circ} \mathrm{C}$ à $5,8^{\circ} \mathrm{C}$, avec des conséquences sur la fréquence et les quantités des précipitations, ainsi que sur l'augmentation des épisodes de sécheresse intense (Altieri et al., 2015). En agriculture, la température et l'hygrométrie sont deux facteurs très importants, c'est pourquoi il est primordial de concevoir des agroécosystèmes résilients face au changement climatique. Il a été démontré que les systèmes ayant un niveau de biodiversité élevé sont des écosystèmes plus résilients aux changements globaux (Méndez et al., 2016). Quel que soit le type de système, plus il est diversifié, plus grande est la variété des niches écologiques, rendant ainsi l'écosystème plus fonctionnel et résilient. Un écosystème pauvre en diversité, tel que des monocultures ou des cultures pérennes (vignes, vergers), résiste moins bien aux aléas climatiques. Un agroécosystème présentant une biodiversité végétale élevée résiste plus efficacement aux événements climatiques pluvieux extrêmes et souffre moins d'évènements physiques tels que les écoulements de boue et les glissements de terrain. Les exploitations pratiquant la monoculture sont très touchées par ces évènements extrêmes (Nicholls et Altieri, 2014). L'augmentation de la biodiversité peut s'envisager localement, mais aussi au niveau du paysage. Une mosaïque paysagère confère à un écosystème une multifonctionnalité au niveau des niches écologiques, et assure ainsi une plus forte résilience aux aléas climatiques (Lempereur et al., 2017). Les îlots de biodiversité aux alentours des parcelles viticoles, comme la mise en place de zones boisées, de prairies et de zones humides, l'installation de refuges à biodiversité tels que des hôtels à insectes, des refuges pour serpents, présentent un bénéfice direct pour la monoculture dans un bassin de production à dominante viticole.

L'utilisation de matériel végétal plus adapté à la sécheresse, notamment des porte-greffes en relation avec les cépages, permet également une meilleure adaptation de la vigne à la contrainte hydrique (INRA et IFV, 2017).

\section{Discussion et conclusion: vers une conception élargie du concept d'agroécologie en viticulture}

\subsection{Une démarche de transition agroécologique au vignoble}

Le modèle agricole productiviste qui s'est développé il y a une soixantaine d'années sous l'impulsion des politiques agricoles avait d'abord eu pour souci de gérer l'autosuffisance alimentaire pour les denrées de première nécessité (céréales, légumes, viandes, lait...), puis la capacité d'exportation des produits agricoles et alimentaires, dont ceux de la filière viticole, contribuant ainsi fortement à l'équilibre de la balance commerciale. Il a désormais dépassé ses limites. Il s'est développé à l'encontre des écosystèmes : sols, eaux de surface et souterraines, biodiversité animale et végétale. Les rendements plafonnent maintenant et les parasites pullulent, conséquence de systèmes trop souvent conduits en monoculture. Dans les pays occidentaux, les conséquences sur la santé humaine, avec l'usage souvent excessif de pesticides et d'autres intrants, provoquent aujourd'hui une opposition grandissante de la société et en particulier des consommateurs, soucieux de leur santé. L'agroécologie promeut au contraire l'accroissement de la production agricole en valorisant les capacités des écosystèmes pour lutter biologiquement contre les ravageurs et les adventices des cultures. En viticulture, les apports de la littérature sont surtout concentrés sur les pratiques agroécologiques prises individuellement; ils permettent in fine de retenir un certain nombre de principes applicables pour orienter les systèmes conventionnels vers une 
démarche de transition agroécologique dans les vignobles (Hazard et al., 2017) :

- la réduction de la pression phytosanitaire par une régulation biologique des bioagresseurs, et les apports de la génétique pour la création de variétés résistantes;

- la nécessité de conserver, voire de reconstituer, un habitat pour les auxiliaires de la vigne et les zones d'intérêt écologiques ;

- le développement de systèmes agroforestiers pour briser la monoculture de la vigne et apporter le bénéfice d'une plantation arborée;

- la conservation de la qualité des sols et leur nutrition par un non-labour et un couvert permanent permettant d'obtenir un niveau satisfaisant de matière organique $(>2 \%)$;

- une diversité végétale à l'échelle des parcelles, mais aussi du paysage pour renforcer la résilience des agroécosystèmes.

\subsection{Conception de systèmes productifs agroécologiques}

Reste alors posée la question de la conception de systèmes productifs agroécologiques dans leur ensemble, de la taille à la récolte, en prenant en compte les contextes de production. Les principes de l'agroécologie appliqués à la viticulture devraient in fine permettre d'introduire de la complexité pour une mise au point et une gestion optimum des relations entre système viticole et écosystèmes naturels. Une transition agroécologique réussie reposerait alors sur l'adoption par les viticulteurs de pratiques co-construites localement, en fonction du contexte et de l'écosystème dans lesquels s'insère l'activité, pour un management optimal des services écosystémiques.

La conception d'agroécosystèmes optimisant les fonctions écosystémiques supportées par la biodiversité est un réel enjeu du point de vue écologique, mais au-delà des performances agronomiques et écologiques de ces systèmes, il s'agit également de prendre en compte les enjeux socio-économiques des exploitations viticoles. Ces systèmes doivent en effet aussi permettre aux producteurs de dégager un revenu pour vivre dignement de leur profession. L'agroécologie porte intrinsèquement l'idée de performance économique, clairement affichée dans le Projet agroécologique pour la France, interrogeant ainsi les coûts et les niveaux de valorisation des pratiques. Dans le contexte actuel, la mise au point de systèmes de production performants, c'est-à-dire qui permettent d'atteindre les rendements visés en minimisant les impacts environnementaux et en préservant la biodiversité tout en répondant aux enjeux socio-économiques, n'a jamais été aussi prégnante. Cependant, la littérature est assez dépourvue d'analyses des performances multiples des agroécosystèmes viticoles. Une évaluation de la multifonctionnalité de ces systèmes, incluant les coûts de production et la valorisation des produits par exemple, permettrait pourtant de préciser les types de systèmes optimaux par rapport aux différents contextes de production, et de mieux prédire l'évolution de leurs performances dans un contexte environnemental changeant. Ainsi, le choix de critères agronomiques, environnementaux et socio-économiques pertinents à partir de cette revue de la littérature devrait permettre, via une analyse multicritère, de construire et d'évaluer des scénarios d'évolution des modes de conduite conventionnels de la vigne vers une démarche agroécologique, afin de replacer l'agronomie, l'écologie et l'humain au centre du système.

\subsection{Une remise en cause des pratiques établies qui génère incertitude et risque}

Le système conventionnel développé depuis plus d'un demi-siècle est basé sur des pratiques bien connues, et notamment sur l'usage systématique d'intrants afin d'assurer une production maximale, sans tenir compte de toute forme de régulation biologique. De ce fait, l'agroécologie amène des principes opposés, avec beaucoup d'incertitude sur la capacité d'application des méthodes et leur efficacité, ce qui génère une prise de risque (Caquet et al., 2020) à l'égard de la production quantitative et qualitative, notamment pour le raisin. Mais ce risque est également bien présent à l'échelle de la filière, qui va devoir assumer une plus grande diversité des producteurs, des produits et des consommateurs, dont les comportements d'acheteur, souvent aléatoires, ne présentent pas un gage de sécurité à l'égard d'un système de production.

Il importe alors d'évaluer l'incertitude et les risques de systèmes nouveaux de production particulièrement complexes, et ce d'autant qu'ils reposent sur des processus biotechniques. L'agroécologie met également en exergue les partages de savoirs et d'expériences dans le cadre de sciences participatives pour enrichir de façon pragmatique les niveaux d'innovation et les applications dans différentes situations (Caquet et al., 2020).

\subsection{Un courant sociétal}

Au-delà d'une approche scientifique et de l'analyse de pratiques agricoles spécifiques, l'agroécologie représente un courant sociétal (Calame, 2016) ayant pour ambition de réconcilier les différents maillons des filières, des producteurs aux consommateurs (Warner, 2007), ainsi que les ruraux et les urbains, pour redynamiser les territoires ruraux subissant exode rural et désertification depuis plusieurs décennies. Les systèmes agroécologiques sont à même d'intégrer les enjeux sociétaux comme le respect de la santé des travailleurs au sein des vignobles et celle des riverains, voire celle des consommateurs de vin, en supprimant l'utilisation des produits chimiques toxiques. Les viticulteurs engagés dans une démarche agroécologique ont ainsi la capacité de créer du lien avec la société, par exemple à travers des évènements de partage au sein du vignoble (visites guidées thématiques : flore et faune locale, agroforesterie, débats sur les relations producteurs-consommateurs). L'agroécologie implique alors un dialogue entre les parties prenantes permettant une valorisation, qu'elle soit monétaire ou non, des services écosystémiques, ce qui milite pour l'intégration des différentes parties prenantes dans les processus de conception et d'évaluation des agroécosystèmes à la manière de Nicholls et al. (2004) et Brévault et Clouvel (2019).

Il apparaît clairement que, si la mise en œuvre de l'agroécologie n'est pas toujours aisée et présente des risques et incertitudes, ses principes sont utiles pour repenser les systèmes viticoles actuels et les accompagner vers plus de durabilité. Cela ne pourra pas se faire sans associer l'ensemble 


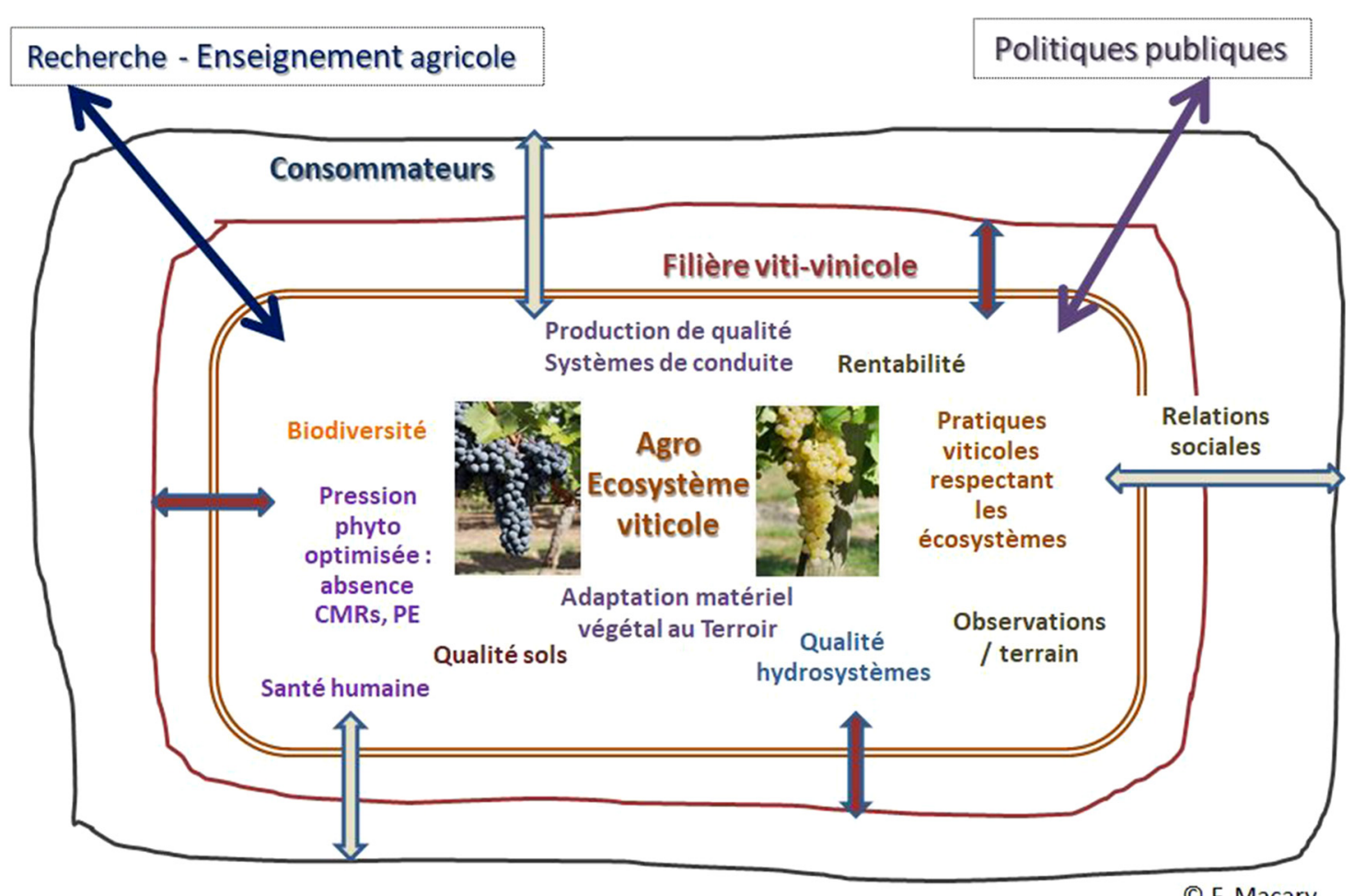

Fig. 6. Synthèse de l'agroécologie en viticulture (F. Macary).

Fig. 6. Synthesis of agroecology in viticulture (F. Macary).

des parties prenantes dans une démarche intégrative (Fig. 6). Il s'agit alors de redéfinir la relation de la viticulture avec la société en s'appuyant sur des recherches participatives, la promotion de la consommation responsable et le renouvellement des politiques publiques nationales et locales.

Remerciements. Ce travail a été réalisé dans le cadre du projet PhytoCOTE avec le soutien financier de l'Agence nationale de la recherche (ANR) dans le cadre du Programme d'investissements d'avenir, au sein du Laboratoire d'excellence COTE (ANR-10-Labex-45), et de la Région Nouvelle-Aquitaine (2015-1R20602). Nous remercions Sybille de Mareschal, documentaliste au centre INRAE de Bordeaux - Cestas pour nous avoir aidés dans les démarches de recherches bibliographiques.

\section{Références}

Alavanja MR, Hoppin JA, Kamel F. 2004. Health effects of chronic pesticide exposure: cancer and neurotoxicity. Public Health 25: 155-197. DOI: 10.1146/annurev.publhealth.25.101802.123020.

Albrecht A, Kandji S. 2003. Carbon sequestration in tropical agroforestry systems. Agriculture, Ecosystems and Environment 99: 15-27. DOI: 10.1016/S0167-8809(03)00138-5.

Altieri MA. 1995. Agroecology: the science of sustainable agriculture. Boulder, CO, USA: Westeview Press, 433 p.

Altieri MA. 1999. The ecological role of biodiversity in agrosystems. Agriculture, Ecosystems and Environment 74: 19-31. DOI: 10.1016/S0167-8809(99)00028-6.

Altieri MA, Nicholls C, Henao A, Lana M. 2015. Agroecology and the design of climate change-resilient farming systems. Agronomy for Sustainable Development 35: 869-890. DOI: 10.1007/ s13593-015-0285-2.

Aubertot JN, Barbier JM, Carpentier A, Gril JJ, Guichard L, Lucas P, et al. 2005. Pesticides, agriculture et environnement. Réduire l'utilisation des pesticides, en limiter les impacts environnementaux. Expertise scientifique collective. Paris, France: INRA, Cemagref, 902 p. HAL Id: hal-02832492, version 1.

Aurias A, Dioux O, Germain M. 2016. Pesticides et santé. Un dossier accablant. La recherche 509: 34-54. https://www.larecherche.fr/ parution/mensuel-509.

Baldi I, Lebailly P. 2007. Cancers et pesticides. La revue du praticien 57(15): 40-44.

Baldi I, Lebailly P, Rondeau V, Bouchard V, Blanc-Lapierre A, Bouvier G. 2012. Levels and determinants of pesticide exposure in operators involved in treatment of vineyards: results of the PESTEXPO study. Journal of Exposure Science and Environmental Epidemiology 22: 593-600. DOI: 10.1038/jes.2012.82.

Baret P. 2017. Un changement de paradigme. Messages du Secours Catholique-Caritas France 720: 16.

Barriuso E, ed. 2004. Estimation des risques environnementaux des pesticides. Paris, France: INRA éditions, 113 p. HAL Id: hal02828881, version 1 .

Baudry J, Bunce RGH, Burel F. 2000. Hedgerows: an international perspective on their origin, function and management. Journal of Environmental Management 60: 7-22. DOI: 10.1006/ jema.2000.0358.

Bourguignon C. 2017. Manifeste pour une agriculture durable. Arles, France: Actes Sud, 80 p.

Brévault T, Clouvel P. 2019. Pest management: reconciling farming practices and natural regulations. Crop Protection 115: 1-6. DOI: 10.1016/j.cropro.2018.09.003. 
Calame M. 2016. Comprendre l'agroécologie. Origines, principes et politiques. Paris, France: Éditions Charles Léopold Mayer. ISBN 978-2-84377- 202-3.

Caquet T, Gascuel C, Tixier-Boichard M, coord. 2020. Agroécologie. Des recherches pour la transition des filières et des territoires. Versailles, France: Quae, 104 p. DOI: 10.35690/978-2-75923130-0.

Cervek C. 2012. Intégrer la biodiversité dans les systèmes d'exploitation agricole. Innovations agronomiques 25: 143-154. https://hal.inrae.fr/hal-02650586.

Côte FX, Poirier-Magona E, Perret S, Rapidel B, Roudier P, Thirion MC, eds. 2018. La transition agroécologique des agricultures du Sud. Versailles, France:Quae, 368 p. DOI: 10.35690/978-2-75922822-5.

Dalgaard T, Hutchings NJ, Porter JR. 2003. Agroecology, scaling and interdisciplinarity. Agriculture Ecosystems \& Environment 100(1): 39-51. DOI: 10.1016/S0167-8809(03)00152-X.

De Schutter O. 2010. Rapport du rapporteur spécial sur le droit à l'alimentation. Nations Unies, Conseil des Droits de l'homme, A/ HRC/16/49, 23 p.

Doré T, Bellon S. 2019. Les mondes de l'agroécologie. Versailles, France: Quae, 176 p. https://www.quae.com/produit/1560/ 9782759230037/les-mondes-de-l-agroecologie.

Doré T, Réchauchère O, Schmidely P. 2008. Les clés des champs. L'agriculture en question. Versailles, France: Quae, 192 p. https:// www.quae.com/produit/952/9782759209699/les-cles-des-champs.

Duru M, Farès M, Therond O. 2014. Un cadre conceptuel pour penser maintenant (et organiser demain) la transition agroécologique de l'agriculture dans les territoires. Cahiers agricultures 23(2): 84-95. DOI: 10.1684 /agr.2014.0691.

FAO. 2018a. FAO'S work on agroecology. A pathway to achieving the SDGs. Rome, Italy: Food and Agriculture Organization of the United Nations.

FAO. 2018b. The 10 elements of agroecology. Guiding the transition to sustainable food and agricultural systems. Rome, Italy: Food and Agriculture Organization of the United Nations.

Francis C, Lieblein G, Gliessman S, Breland TA, Creamer N, Harwood R, et al. 2003. Agroecology: the ecology of food systems. Journal of Sustainable Agriculture 22: 99-118. DOI: 10.1300/J064v22n03_10.

Garcia L, Damour G, Gary C, Follain S, Le Bissonnais Y, Metay A. 2019. Trait-based approach for agroecology: contribution of service crop root traits to explain soil aggregate stability in vineyards. Plant and Soil 435(1-2): 1-14. DOI: 10.1007/ s11104-018-3874-4.

Garrigou A, Laurent C, Berthet A, Colosio C, Jas N, DaubasLetourneux V, et al. 2020. Critical review of the role of PPE in the prevention of risks related to agricultural pesticide use. Safety Science 123. DOI: 10.1016/j.ssci.2019.104527.

Gary C, Mézière D, Barbier JM, Bernos L, Clément C, Constant N, et al. 2009. Analyse comparative de différents systèmes en viticulture. In: ECOPHYTO R\&D : vers des systèmes de culture économes en produits phytosanitaires. Rapport d'expertise. Paris, France: Inra éditions, tome III, $57 \mathrm{p}+$ annexes.

Gary C, Metral R, Metay A, Garcia L, Merot A, Smits N, et al. 2017. Towards an agroecological viticulture: advances and challenges. In: Proceedings of the 20th GiESCO International Meeting, November 5-10, 2017, Mendoza, Argentina. https://hal.archivesouvertes.fr/hal-01664474.

Gliessman SR. 1998. Agroecology: ecological processes in sustainable agriculture. Chelsea, UK: Ann Arbor.

Gliessman SR. 2015. Agroecology: the ecology of sustainable food systems. London, UK: Boca Raton.
Hazard L, Magrini MB, Martin G. 2017. Transition agroécologique : définition. In: Dictionnaire d'agroecologie. https://dicoagroecolo gie.fr/encyclopedie/transition-agroecologique/.

Hazard L, Monteil C, Duru M, Bedoussac L, Justes E, Theau JP. 2016. Agroécologie : définition. In: Dictionnaire d'agroecologie. https:// dicoagroecologie.fr/encyclopedie/agroecologie/.

Hatt S, Artru S, Brédart D, Lassois L, Francis F, Haubruge E, et al. 2016. Towards sustainable food systems: the concept of agroecology and how it questions current research practices, a review. Biotechnologie, Agronomie, Société et Environnement 20 (S1): 10. DOI: 10.25518/1780-4507.12997.

Hénin S. 1967. Les acquisitions techniques en production végétale et leurs applications. Économie rurale 74: 37-44. DOI: 10.3406/ ecoru.1967.1989.

Hénin S. 1980. Rapport du groupe de travail activités agricoles et qualité des eaux - Tome II - sous groupes : effluents d'élevage, produits phytosanitaires, systèmes de cultures. Paris, France: ministère de l'Agriculture et ministère de l'Environnement et du Cadre de vie, 294 p.

Henneron L. et al. 2015. Fourteen years of evidence for positive effects of conservation agriculture and organic farming on soil life. Agronomy for Sustainable Development 35(1): 169-181. DOI: 10.1007/s13593-014-0215-8.

INRA, IFV. 2017a. Guide de l'agroécologie en viticulture. Développement des mesures agroenvironnementales. Paris, France: INAO, IFV, 52 p. https:/www.vignevin.com/outils/ guide-agroecologique/.

INRA, IFV. 2017b. Pour une viticulture durable et de qualité: les résistances au cœur de la sélection variétale, Dossier de presse. Paris, France: INRA, IFV, 22 p. http://observatoire-cepagesresistants.fr/wp-content/uploads/2017/06/resistance-varietales-dos sier_presse_janv2017.pdf.

INRA, IFV, Chambres d'agriculture. 2011. Guide CEPVITI - Coconception de systèmes viticoles économes en produits phytosanitaires, Ecophyto2018. Paris, France: INRA, IFV, Chambres d'agriculture, 28 p. https://www.vignevin.com/reductions-intrants/ systemes-viticoles-economes-en-intrants/.

INSERM. 2013. Pesticides: effets sur la santé. Rapport. Paris, France: INSERM, XII-1001 p. http://www.ipubli.inserm.fr/handle/10608/ 4819.

JORF. 2014. Loi n ${ }^{\circ}$ 2014-1170 du 13 octobre 2014 d'avenir pour l'agriculture, l'alimentation et la forêt, Paris.

Lempereur V, Gautier J, Herbin C. 2017. Le guide de l'agroécologie en viticulture, un outil pour la filière. In: BIO Web Conferences 9 , 40th World Congress of Vine and Wine. DOI: 10.1051/bioconf/ 20170901007.

Letourneau D, Jedlicka J, Bothwell S, Moreno C. 2009. Effects of natural enemy biodiversity on the suppression of arthropod herbivores in terrestrial ecosystems. Ecology, Evolution and Systematics 40: 573-592. DOI: 10.1146/annurev.ecol sys. 110308.120320 .

MAAF. 2012. Agricultures, produisons autrement: projet agroécologique pour la France. Paris, France: ministère en charge de l'Agriculture, 16 p. https://agriculture.gouv.fr/le-projet-agro-ecolo gique-en-france.

Méndez VE, Bacon CM, Cohen R, Gliessman SR. 2016. Agroecology: a transdisciplinary, participatory and action-oriented approach. London, UK: Boca Raton, 284 p. DOI: 10.1201/b19500.

Mulumba L, Lal R. 2008. Mulching effects on selected soil physical properties. Soil \& Tillage Research 98: 106-111. DOI: 10.1007/ 978-90-481-3585-1 275.

Nicholls C, Altieri M, Dezanet A, Lana M, Feistauer D, Ouriques M. 2004. A rapid, farmer-friendly agroecological method to estimate 
soil quality and crop health in vineyard systems. Biodynamics 33-40. http://agroecology.pbworks.com/f/biodyn-indicators.pdf.

Nicholls C, Altieri M. 2014. Agroecology: designing climate change resilient small farming systems in the developing world. In: Agroecology for food security and nutrition, Proceedings of the FAO International Symposium, Rome, Italy, pp. 271-295. DOI: 10.1007/s13593-015-0285-2.

Parmentier B. 2007. Nourrir l'humanité. Les grands problèmes de l'agriculture mondiale au $\mathrm{XXI}^{\mathrm{e}}$ siècle. Paris, France: La Découverte, 294 p. https://editionsladecouverte.fr/catalogue/ index-Nourrir 1 humanit -9782707157027.html.

Rahbi P. 2015. L'agroécologie, une éthique de vie. Arles, France: Actes Sud, $80 \mathrm{p}$.

Rennings K. 2000. Redefining innovation - eco-innovation research and the contribution from ecological economics. Ecological Economics 32: 319-332. DOI: 10.1016/S0921-8009(99)00112-3.

Schaller N. 2013. L'agriculture de conservation. Paris, France: ministère de l'Agriculture, de l'Agroalimentaire et de la Forêt, Centre d'études et de prospective, 61, 4 p. DOI: 10.13140/2.1.1359.5202.

Shields MW, Tompkins JM, Saville DJ, Meurk CD, Wratten S. 2016. Potential ecosystem service delivery by endemic plants in New
Zealand vineyards: successes and prospects. Peer J, Life \& Environment. DOI: 10.7287/peerj.preprints.1995.

Tardieu V. 2012. Vive l'agro-révolution française! Paris, France: Belin, 464 p, ISBN: 978-2-7011-5973-7.

Tisseyre B, Ojeda H, Taylor J. 2007. New technologies and methodologies for site-specific viticulture. International Journal of Wine and Vine Research 41(2): 63-76. DOI: 10.20870/oenoone.2007.41.2.852.

Warner KD. 2007. The quality of sustainability: agroecological partnerships and the geographic branding of California winegrapes. Journal of Rural Studies 23: 142-155. DOI: 10.1016/j.jrurs tud.2006.09.009.

Wezel A, Bellon S, Doré T, Francis C, Vallod D, David C. 2009. Agroecology as a science, a movement and a practice. A review. Agronomy for Sustainable Development 29: 503-515. DOI: 10.1051/agro/2009004.

Wezel A, Casagrande M, Celette F, Vian JF, Ferrer A, Peigné J. 2014. Agroecological practices for sustainable agriculture. A review. Agronomy for Sustainable Development 34: 1-20. DOI: 10.1007/ s13593-013-0180-7.

Citation de l'article : Macary F, Guerendel F, Alonso Ugaglia A. 2020. Quels apports de la littérature pour comprendre et construire la transition agroécologique en viticulture? Cah. Agric. 29: 38. 